\title{
Effective control of SARS-CoV-2 transmission between healthcare workers during a period of diminished community prevalence of COVID-19
}

\author{
Nick K Jones ${ }^{1}$ \\ ${ }^{1}$ University of Cambridge
}

June 16, 2020

\begin{abstract}
We previously demonstrated that 31/1,032 (3\%) asymptomatic healthcare workers (HCW) from a large teaching hospital in Cambridge UK tested positive for SARS-CoV-2 in April 2020. ${ }^{1}$ 26/169 (15\%) HCWs with symptoms of coronavirus disease 2019 (COVID-19) also tested positive. Here, we report on our ongoing studies, and provide a temporal analysis of SARS-CoV-2 infection rates during the ongoing UK 'lockdown'. Corresponding with a decline in patient admissions with COVID-19, the proportion of both asymptomatic and symptomatic HCWs testing positive rapidly declined to near-zero between 25th April and 24th May 2020. These data demonstrate how infection prevention and control measures including staff testing may help prevent hospitals from becoming independent 'hubs' of SARS-CoV-2 transmission, and illustrate how, with appropriate precautions, organisations in other sectors may be able to resume on-site work safely.
\end{abstract}

Effective control of SARS-CoV-2 transmission between healthcare workers during a period of diminished community prevalence of COVID-19

Nick K. Jones ${ }^{1,2,3,4 *}$, Lucy Rivett ${ }^{1,2 *}$, Dominic Sparkes ${ }^{1,2 *}$, Sally Forrest ${ }^{3,4 *}$, Sushmita Sridhar ${ }^{3,4,5}$, Jamie Young $^{6}$, Joana Pereira-Dias ${ }^{3,4}$, Claire Cormie ${ }^{3,4}$, Harmeet Gill ${ }^{3,4}$, Nicola Reynolds ${ }^{7}$, Michelle Wantoch ${ }^{8,9}$, Matthew Routledge ${ }^{1,2}$, Ben Warne ${ }^{1,4}$, Jack Levy ${ }^{10}$, William David Cordova Jiménez ${ }^{10}$, Fatima N. B. Samad $^{10}$, Chris McNicholas ${ }^{11}$, Mark Ferris ${ }^{12}$, Jane Gray ${ }^{13}$, Michael Gill ${ }^{13}$, The CITIID-NIHR COVID19 BioResource Collaboration, Martin Curran ${ }^{2}$, Stewart Fuller ${ }^{14}$, Afzal Chaudhry ${ }^{15}$, Ashley Shaw ${ }^{15}$, John R. Bradley ${ }^{3,16}$, Greg Hannon ${ }^{13}$, Ian Goodfellow ${ }^{17}$, Gordon Dougan ${ }^{3,4}$, Kenneth G.C. Smith ${ }^{3,4}$, Paul J.

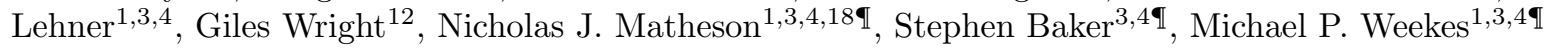

Correspondence: Dr. Michael Weekes, mpw1001@cam.ac.uk

*Joint first authorship

IJoint last authorship

1 Department of Infectious Diseases, Cambridge University NHS Hospitals Foundation Trust, Cambridge, UK

2 Clinical Microbiology \& Public Health Laboratory, Public Health England, Cambridge, UK

3 Department of Medicine, University of Cambridge, Cambridge, UK 
4 Cambridge Institute of Therapeutic Immunology \& Infectious Disease (CITIID), Jeffrey Cheah Biomedical Centre, Cambridge Biomedical Campus, University of Cambridge, Cambridge, UK

5 Wellcome Sanger Institute, Hinxton, UK

6 Academic Department of Medical Genetics, University of Cambridge, Cambridge, UK

7 Wellcome-MRC Cambridge Stem Cell Institute, Jeffrey Cheah Biomedical Centre, Cambridge Biomedical Campus, University of Cambridge, Cambridge, UK

8 Wellcome - MRC Cambridge Stem Cell Institute, University of Cambridge, Cambridge, UK

9 Department of Haematology, School of Clinical Medicine, University of Cambridge

10 Institute for Manufacturing, Department of Engineering, University of Cambridge, Cambridge, UK

11 Improvement and Transformation Team, Cambridge University Hospitals NHS Foundation Trust, Cambridge, UK

12 Occupational Health and Wellbeing, Cambridge University Hospitals NHS Foundation Trust, Cambridge, $\mathrm{UK}$

13 Cancer Research UK Cambridge Institute, University of Cambridge, Cambridge, UK

14 National Institutes for Health Research Cambridge, Clinical Research Facility, Cambridge, UK

15 Cambridge University Hospitals NHS Foundation Trust, Cambridge, UK

16 National Institutes for Health Research Cambridge Biomedical Research Centre, Cambridge, UK

17 Division of Virology, Department of Pathology, University of Cambridge, Cambridge, UK

18 NHS Blood and Transplant, Cambridge, UK

\section{The CITIID-NIHR COVID-19 BioResource Collaboration}

Principal Investigators: Stephen Baker, John Bradley, Gordon Dougan, Ian Goodfellow, Ravi Gupta, Paul J. Lehner, Paul A. Lyons, Nicholas J. Matheson, Kenneth G.C. Smith, M. Estee Torok, Mark Toshner, Michael P. Weekes

Infectious Diseases Department: Nick K. Jones, Lucy Rivett, Matthew Routledge, Dominic Sparkes, Ben Warne

SARS-CoV-2 testing team: Claire Cormie, Sally Forrest, Harmeet Gill, Iain Kean, Joana Pereira-Dias, Nicola Reynolds, Sushmita Sridhar, Michelle Wantoch, Jamie Young

COG-UK Cambridge Sequencing Team: Sarah Caddy, Laura Caller, Theresa Feltwell, Grant Hall, William Hamilton, Myra Hosmillo, Charlotte Houldcroft, Aminu Jahun, Fahad Khokhar, Luke Meredith, Anna Yakovleva

NIHR BioResource: Helen Butcher, Daniela Caputo, Debra Clapham-Riley, Helen Dolling, Anita Furlong, Barbara Graves, Emma Le Gresley, Nathalie Kingston, Sofia Papadia, Hannah Stark, Kathleen E. Stirrups, Jennifer Webster

Research nurses: Joanna Calder, Julie Harris, Sarah Hewitt, Jane Kennet, Anne Meadows, Rebecca Rastall, Criona O,Brien, Jo Price, Cherry Publico, Jane Rowlands, Valentina Ruffolo, Hugo Tordesillas

CRUK: Michael Gill, Jane Gray, Greg Hannon 
NIHR Cambridge Clinical Research Facility: Karen Brookes, Laura Canna, Isabel Cruz, Katie Dempsey, Anne Elmer, Naidine Escoffery, Stewart Fuller, Heather Jones, Carla Ribeiro, Caroline Saunders, Angela Wright

Cambridge Cancer Trial Centre: Rutendo Nyagumbo, Anne Roberts

Clinical Research Network Eastern: Ashlea Bucke, Simone Hargreaves, Danielle Johnson, Aileen Narcorda, Debbie Read, Christian Sparke, Lucy Worboys

Administrative staff, CUHNFT: Kirsty Lagadu, Lenette Mactavous

CUHNFT NHS Foundation Trust: Tim Gould, Tim Raine, Ashley Shaw

Cambridge Cancer Trials Centre: Claire Mather, Nicola Ramenatte, Anne-Laure Vallier

Legal/Ethics: Mary Kasanicki

CUHNFT Improvement and Transformation Team: Penelope-Jane Eames, Chris McNicholas, Lisa Thake

Clinical Microbiology \& Public Health Laboratory (PHE): Neil Bartholomew, Nick Brown, Martin Curran, Surendra Parmar, Hongyi Zhang

Occupational Health: Ailsa Bowring, Mark Ferris, Geraldine Martell, Natalie Quinnell, Giles Wright, Jo Wright

Health and Safety: Helen Murphy

Department of Medicine Sample Logistics: Benjamin J. Dunmore, Ekaterina Legchenko, Stefan Gräf, Christopher Huang, Josh Hodgson, Kelvin Hunter, Jennifer Martin, Federica Mescia, Ciara O'Donnell, Linda Pointon, Joy Shih, Rachel Sutcliffe, Tobias Tilly, Zhen Tong, Carmen Treacy, Jennifer Wood

Department of Medicine Sample Processing and Acquisition: Laura Bergamaschi, Ariana Betancourt, Georgie Bowyer, Aloka De Sa, Maddie Epping, Andrew Hinch, Oisin Huhn, Isobel Jarvis, Daniel Lewis, Joe Marsden, Simon McCallum, Francescsa Nice, Ommar Omarjee, Marianne Perera, Nika Romashova, Mateusz Strezlecki, Natalia Savoinykh Yarkoni, Lori Turner

Epic team/other computing support: Barrie Bailey, Afzal Chaudhry, Rachel Doughton, Chris Workman Statistics/modelling: Caroline Trotter

Department of Engineering: William David Cordova Jiménez, Jack Levy, Fatima N. B. Samad

\section{Abstract}

We previously demonstrated that 31/1,032 (3\%) asymptomatic healthcare workers (HCW) from a large teaching hospital in Cambridge UK tested positive for SARS-CoV-2 in April 2020. ${ }^{1}$ 26/169 (15\%) HCWs with symptoms of coronavirus disease 2019 (COVID-19) also tested positive. Here, we report on our ongoing studies, and provide a temporal analysis of SARS-CoV-2 infection rates during the ongoing UK 'lockdown'. Corresponding with a decline in patient admissions with COVID-19, the proportion of both asymptomatic and symptomatic HCWs testing positive rapidly declined to near-zero between $25^{\text {th }}$ April and $24^{\text {th }}$ May 2020. These data demonstrate how infection prevention and control measures including staff testing may help prevent hospitals from becoming independent 'hubs' of SARS-CoV-2 transmission, and illustrate how, with appropriate precautions, organisations in other sectors may be able to resume on-site work safely.

Introduction 
The role of nosocomial transmission of SARS-CoV-2 has been highlighted by recent evidence suggesting that $20 \%$ of SARS-CoV-2 infections among UK hospital patients and up to $89 \%$ of infections among HCWs may have originated in hospital. ${ }^{2,3}$ Since the introduction of 'lockdown' in the UK, community transmission rates of SARS-CoV-2 have generally declined. ${ }^{4}$ Conversely, concerns have been raised that hospitals could become independent 'hubs' for ongoing SARS-CoV-2 transmission between patients and HCWs, which would effectively prolong the epidemic. ${ }^{3}$ In this context, evolution of the epidemic curves of a hospital's symptomatic and asymptomatic workforce have not been well described.

We recently initiated a comprehensive HCW screening programme for SARS-CoV-2 in a large teaching hospital in Cambridge, UK. Over a three-week period from $6^{\text {th }}$ to $24^{\text {th }}$ April $2020,3 \%(31 / 1,032)$ HCWs in the asymptomatic screening arm , $15.4 \%$ (26/169) HCWs in the symptomatic screening arm and $7.7 \%$ $(4 / 52)$ contacts in the symptomatic household contact screening arm tested positive for SARS-CoV-2. ${ }^{1}$ Our data from the asymptomatic screening arm were consistent with another study since published. ${ }^{5}$ Over the subsequent four weeks from $25^{\text {th }}$ April to $24^{\text {th }}$ May 2020, we performed a further 3,388 additional tests. Here, we present these longitudinal data, in the context of the hospital patient population and wider local community.

\section{Results}

Testing for SARS-CoV-2 RNA was performed by real time RT-PCR on throat and nose swab samples taken from HCWs from Cambridge University Hospitals NHS Foundation Trust (CUHNFT) and their symptomatic household contacts. Over the new study period (25 ${ }^{\text {th }}$ April 2020 to $24^{\text {th }}$ May 2020), 2,611 additional tests were performed in the $H C W$ asymptomatic screening arm, 555 additional tests in the HCW symptomatic screening arm and 216 additional tests in the $H C W$ household contact screening arm. A further six tests did not have a clearly recorded arm of origin. Over the entire study period, the median age of HCWs was 36.5 years, and 35.5 years for their household contacts. $68.4 \%$ were female and $31.6 \%$ were male. Of individuals testing positive over the whole study period, the median age of HCWs was 32 and 47 years for their household contacts. $77.9 \%$ of all positive tests were from females and $22.1 \%$ from males. Table 1 summarises the total number of HCWs testing positive through either arm of the screening programme, according to job role. Comparison of the proportions of hospital employees from each job role that tested positive through the $H C W$ symptomatic screening arm revealed no statistically significant difference (Pearson's chi-square test $\mathrm{p}=0.419)$. Reasonable comparison of the proportions testing positive through the HCW asymptomatic screening arm was not possible due to non-random sampling of different areas of the hospital, meaning some job roles had been more frequently targeted for asymptomatic screening than others.

\begin{tabular}{llll}
\hline Role & HCW asymptomatic screening arm & HCW symptomatic screening arm & Total number of hos \\
\hline Nurse & 25 & 19 & 3621 \\
Healthcare assistant & 14 & 8 & 1734 \\
Doctor & 8 & 6 & 1871 \\
Cleaners & 2 & 3 & 560 \\
Radiographer & 2 & 1 & 217 \\
Radiology support worker & 0 & 1 & 35 \\
Physiotherapist & 1 & 0 & 116 \\
\hline
\end{tabular}

Table 1. Combined data for SARS-CoV-2 RNA positive HCWs by role and screening arm, from the present study and our previous study ${ }^{1}$. Difference in proportions of HCWs testing positive through the symptomatic screening arm was analysed using Pearson's chi-square test.

Overall, 360 individuals underwent repeat testing, either as part of the asymptomatic screening programme, 
or for other reasons as previously described. ${ }^{1}$ Median turnaround time from sample arrival in the laboratory to final verification was 18 hours 45 mins. Positive results were called out on the same day, with negative results emailed within 24 hours.

Between $25^{\text {th }}$ April 2020 and $24^{\text {th }}$ May 2020, a total of 34 new positive tests were reported. In the $H C W$ symptomatic and HCW symptomatic household contact screening armscombined (reflecting all individuals with self-reported symptoms at the time of testing), 13/771 (1.7\%) tests were positive, which was significantly lower than $30 / 221(13 \%)$ in the original study period (Fisher's exact test $\mathrm{p}<0.0001$ ). In the $H C W$ asymptomatic screening arm, 21/2,611 (0.8\%) tests were positive, which again was significantly lower than $31 / 1,032(3 \%)$ in the original study period (Fisher's exact test $\mathrm{p}<0.0001$ ). As we previously observed ${ }^{1}$, individuals captured in the $H C W$ asymptomatic screening arm were generally asymptomatic at the time of screening, however these individuals could be divided into sub-groups. In the first subgroup, 8/21 (38\%) HCWs had no symptoms at all. Of these, 5/8 (63\%) remained entirely asymptomatic 5-7 weeks after their positive test, whereas $2 / 8(25 \%)$ developed symptoms $24-48 \mathrm{~h}$ after testing. One HCW could not be contacted to obtain further history. In the second subgroup, 6/21 (29\%) had retrospectively experienced some symptoms prior to screening. Of these, $5 / 6(83 \%)$ had symptoms with a high pre-test probability of COVID- $19^{1}$ commencing $>7$ days prior to screening, of whom $3 / 5$ had appropriately self-isolated then returned to work, and $1 / 5$ was tested shortly after developing symptoms. $1 / 6(17 \%)$ had symptoms with a low pre-test probability of COVID- $19^{1}$ commencing $<7$ days prior to screening and had not self-isolated. In the third subgroup, $7 / 21(33 \%)$ were detected through repeat sampling of HCW who previously tested positive. Of these, $4 / 7(57 \%)$ were tested to determine their suitability to return to work with severely immunocompromised / immunosuppressed patients, as dictated by UK national guidance. ${ }^{6}$ The remaining $3 / 7(43 \%)$ were from HCWs tested incidentally for a second time in the asymptomatic HCW screening programme. The median interval between serial positive tests was 16.5 days (IQR 9.5-19.5). All cases were attributable to prolonged SARS-CoV-2 RNA detection from a single infection, rather than re-infection. Our approach to patients with repeatedly positive SARS-CoV-2 PCR tests is described in the Methods.

The fraction of positive tests amongst the HCW asymptomatic, and HCW symptomatic and household contact screening groups combined varied over time (Figure 1A, Table 2). In particular, during the last two weeks of the study period (11 ${ }^{\text {th }}$ May to $24^{\text {th }}$ May) we identified only 4 positive SARS-CoV-2 samples from 2,016 tests performed, 2 from the $H C W$ asymptomaticand 2 from the HCW symptomatic / symptomatic household contact arms. This fall in positive HCW tests mirrored the decline in both patients testing positive at CUHNFT and those tested throughout the wider region (Figure 1B). Similar trends were observed in a smaller cohort study of HCWs in London. ${ }^{7}$
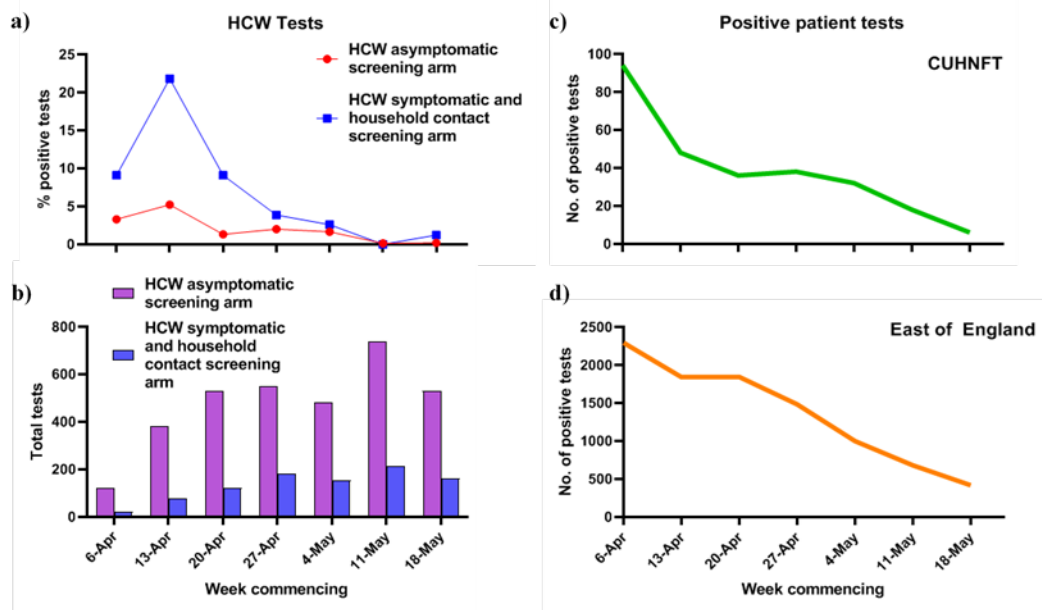
Figure 1: (a) Positive SARS-CoV-2 tests for asymptomatic and symptomatic screening arms by week.(b) Total HCW SARS-CoV-2 tests in CUHNFT performed by week.(c) Total positive SARS-CoV-2 patient tests in Cambridge University Hospital NHS Foundation Trust (CUHNFT) by week. (d)Total positive SARS-CoV-2 tests in the East of England (EOE) by week.

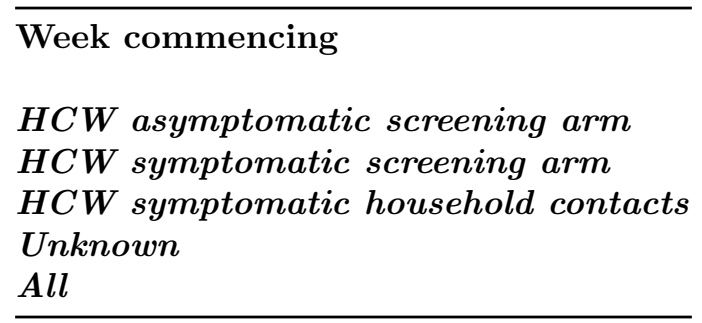

Table 2. Positive tests and total number of SARS-CoV-2 tests performed in each screening arm categorised according to week since starting the healthcare worker testing programme ( $6^{\text {th }}$ April-24 ${ }^{\text {th }}$ May 2020).

In our original study between $6^{\text {th }}$ to $24^{\text {th }}$ April 2020, we described in detail two clusters of HCW infections. ${ }^{1}$ From $25^{\text {th }}$ April 2020 to $24^{\text {th }}$ May 2020, we detected one additional cluster on a general medical ward with a separated area for patients with proven COVID-19 and another for those without. This was identified through targeted screening of the ward over a 24-hour period from $4^{\text {th }}$ to $5^{\text {th }}$ May 2020, in response to four staff testing positive through the $H C W$ symptomatic arm of the screening programme from $27^{\text {th }}$ to $30^{\text {th }}$ April 2020. Reactive screening of a further 40 staff from the same ward identified a further three positive asymptomatic HCWs. In addition, a further two HCWs tested positive in an asymptomatic screen of 30 individuals from a closely related clinical area (designated for non-COVID patients) on $6^{\text {th }}$ May 2020.

\section{Discussion}

Our data demonstrate a dramatic fall in the prevalence of symptomatic and asymptomatic SARS-CoV-2 infection amongst HCWs in our hospital during the study period. On average, the number of secondary infections amongst HCWs arising from each infected HCW (effectively, the reproduction number (R) for SARS-CoV-2 transmission between HCWs) must therefore be $<1$.

As well as acquisition from other HCWs, infections amongst HCWs may also be acquired from patients, as well as other individuals outside the hospital. Our study period coincided with a decline in the rate of infection across our local community, and our data are consistent with a reduction in transmission within the hospital, a reduction in community-based acquisition of infection by HCWs, or (most likely) a combination of both. In the absence of detailed epidemiological data, it is not possible to formally differentiate between these possibilities or determine their relative effect sizes. Nonetheless, our identification of HCW infection clusters in specific areas of the hospital highlighted the potential for workplace acquisition of SARS-CoV-2, which may lead to self-sustaining outbreaks if left uninterrupted. ${ }^{1,8}$ For each of these clusters, timely identification of HCW infection proved effective in terminating chains of hospital transmission between staff, preventing ongoing nosocomial infection.

With the incidence of infection having fallen significantly in hospitalised patients, HCWs and the wider community, many hospitals across the UK and further afield have been afforded precious time to build the infrastructure necessary to establish comprehensive screening programmes in anticipation of a possible second epidemic peak. For hospitals already operating newly established screening programmes, the challenge now is to up-scale to the point that screening can occur at a frequency that permits pre-symptomatic capture of as close to $100 \%$ of all new infections as possible. This approach will enable staff to be removed 
from the workplace at the time of peak infectivity. ${ }^{9}$ The minimum screening frequency required needs to be carefully modelled, with recent estimates suggesting the need for weekly testing to prevent $16-33 \%$ of onward transmission from HCWs, depending on the time taken for results to be reported, and another study estimating the need for daily screening to prevent $65 \%$ of HCW-to-HCW transmission events. ${ }^{2,10}$ In practice, we have observed good results in our hospital with a current frequency of asymptomatic screening every 2-4 weeks. Those being screened are prioritised by anticipated ward-based exposure to COVID-19, with additional targeted screens triggered by excess staff sickness or the identification of symptomatic cases on specific wards. ${ }^{1}$ In addition to asymptomatic screening, testing of symptomatic HCWs is essential for preventing excessive erosion of the hospital workforce by self-isolation on the basis of symptoms alone, and testing of symptomatic HCW household contacts negates the need for unnecessary self-quarantine periods for co-habiting HCWs. We found uptake to the HCW symptomatic household contact screening arm of our programme to be notably lower than the HCW symptomatic arm despite regular communications to advertise the service within CUHNFT. This lack of uptake may reflect a lack of awareness that symptomatic non-HCWs were eligible for testing, provided they shared a household with a hospital employee. Many non-hospital employees may also have been more inclined to attend national testing centres or be less aware of the spectrum of COVID-19 symptoms.

Importantly, our data demonstrate that CUHNFT was not acting as an independent 'hub' for ongoing COVID-19 transmission among HCWs. The absence of nosocomial transmission likely reflects the combined efficacy of HCW testing, stringent prospective and reactive infection prevention and control measures, and appropriate social distancing amongst the workforce. These findings should give reassurance to both hospital staff and patients that healthcare facilities remain safe places to give and receive care. Furthermore, since CUHNFT, with approximately 11,000 staff members (many of whom are based in the hospital) is a major regional employer, we predict that comparable organisations in other sectors may also be able to resume on-site work safely by instigating similar precautions.

\section{Materials and methods}

\section{Staff screening protocols}

We previously described protocols for staff screening, sample collection, laboratory processing and results reporting in detail. ${ }^{1}$ These methods remained unchanged throughout this study period. Two parallel streams of entry into the testing programme included (i) HCW symptomatic, and $\mathrm{HCW}$ symptomatic household contact screening arms and (ii) an $\mathrm{HCW}$ asymptomatic screening arm. In the former, any patient-facing or non-patient-facing HCW could voluntarily refer themselves or a household contact, should they develop symptoms suggestive of COVID-19. In the latter, HCWs could volunteer to take part in a rolling programme of testing for all patient-facing and non-patient-facing staff working in defined clinical areas thought to be at risk of SARS-CoV-2 transmission. Testing was performed (i) at temporary on-site 'Pods'; (ii) via selfswabbing kits delivered to HCWs in their area of work. All individuals in each arm of the programme performed a self-swab at the back of the throat then the nasal cavity, followed by RNA extraction and amplification using real-time RT-PCR. ${ }^{11}$ Cluster investigation was initiated when three or more HCWs working in the same clinical area tested positive for SARS-CoV-2 in a one week period.

\section{Management of $\mathrm{HCW}$ with repeat positive tests}

Current National Institute for Health and Care Excellence (NICE) guidelines require a negative test prior to returning to work with immunocompromised patients. ${ }^{6}$ In accordance with UK national guidance, individuals with repeat positive screens following a minimum period of seven days self-isolation were advised to continue working if they were not scheduled to come into close contact with heavily immunocompromised patients, provided they remained asymptomatic ${ }^{12}$. This approach to managing repeat positive screens is further supported by recent data from the Korea Centers for Disease Control \& Prevention, which showed no clear evidence of onward transmission to the contacts of 285 repeat-positive individuals, 108 of whom had samples taken for attempted viral culture, which was universally unsuccessful. ${ }^{13}$ Additional small studies have also 
demonstrated inability to culture virus from clinical samples obtained later than 8 days after symptom onset, suggesting prolonged detection of viral RNA is unlikely to indicate ongoing risk of transmission. ${ }^{14,15}$

Data extraction and analysis

Swab result data for HCWs and patients were extracted directly from the hospital-laboratory interface software, Epic (Verona, Wisconsin, USA) and from SARS-CoV-2 point of care testing. Data for SARS-CoV2 infections from the local community were extracted from Public Health England's Data Dashboard. ${ }^{4}$ Data were collated using Microsoft Excel, and figures produced with GraphPad Prism (GraphPad Software, La Jolla, California, USA). Fisher's exact test was used to compare the proportion of HCWs testing positive in this study period to that of our previous study period ${ }^{1}$. Pearson's Chi-square test was used for comparison of the proportions of HCWs testing positive in each job role.

Ethics and consent:

As a study of healthcare-associated infections, this investigation is exempt from requiring ethical approval under Section 251 of the NHS Act 2006 (see also the NHS Health Research Authority algorithm, available at http://www.hra-decisiontools.org.uk/research/, which concludes that no formal ethical approval is required). Our study was performed as a service evaluation of the CUHNFT screening programme. The service provided was not changed in any way in order to undertake this evaluation.

\section{Funding}

No funding sources have had any role in data collection, analysis, interpretation, writing of the manuscript or the decision to submit for publication. No authors have been paid to write this article by a pharmaceutical company or any other agency. NKJ, LR, DS, SB, MPW had access to all the data. SB, MPW held final responsibility for the decision to submit the manuscript for publication.

\section{Acknowledgements}

This work was supported by the Wellcome Trust Senior Research Fellowships 108070/Z/15/Z to MPW, 215515/Z/19/Z to SGB and 207498/Z/17/Z to IGG; Collaborative award 206298/B/17/Z to IGG; Principal Research Fellowship 210688/Z/18/Z to PJL; Investigator Award 200871/Z/16/Z to KGCS; Addenbrooke's Charitable Trust (to MPW, SGB, and PJL); the Medical Research Council (CSF MR/P008801/1 to NJM); NHS Blood and Transfusion (WPA15-02 to NJM); National Institute for Health Research (Cambridge Biomedical Research Centre at CUHNFT), to JRB, AC and GD, Cancer Research UK (PRECISION Grand Challenge C38317/A24043 award to JY).

Conflict of Interest statements

Nick Jones has nothing to disclose.

Lucy Rivett has nothing to disclose.

Dominic Sparkes has nothing to disclose.

Sushmita Sridhar has nothing to disclose.

Sally Forrest has nothing to disclose.

Jamie Young has nothing to disclose.

Joana Pereira-Dias has nothing to disclose.

Claire Cormie has nothing to disclose.

Harmeet Gill has nothing to disclose.

Nicola Reynolds has nothing to disclose.

Michelle Wantoch has nothing to disclose. 
Matthew Routledge has nothing to disclose.

Ben Warne reports grants from National Institute for Health Research Cambridge Biomedical Research Centre at the Cambridge University Hospitals NHS Foundation Trust, during the conduct of the study.

Jack Levy has nothing to disclose.

William David Cordova Jiménez has nothing to disclose.

Fatima N. B. Samad has nothing to disclose.

Chris McNicholas has nothing to disclose.

Mark Ferris has nothing to disclose.

Jane Gray has nothing to disclose.

Michael Gill has nothing to disclose.

Martin Curran has nothing to disclose.

Stewart Fuller has nothing to disclose.

Afzal Chaudhry reports grants from Cambridge Biomedical Research Centre at CUHNFT, during the conduct of the study.

Ashley Shaw has nothing to disclose.

John Bradley has nothing to disclose.

Greg Hannon has nothing to disclose.

Ian Goodfellow reports grants from Wellcome Trust (Senior Research Fellowships), grants from Wellcome Trust (Collaborative Award), grants from Addenbrooke's Charitable Trust, during the conduct of the study.

Gordon Dougan reports grants from NIHR, during the conduct of the study.

Kenneth G.C. Smith reports grants from Wellcome Trust, during the conduct of the study.

Paul J. Lehner reports grants from Wellcome Trust Principal Research Fellowship, grants from Addenbrooke's Charitable Trust, during the conduct of the study.

Nicholas J. Matheson reports grants from Medical Research Council (Clinician Scientist Fellowship), grants from NHS Blood and Transfusion, during the conduct of the study.

Giles Wright has nothing to disclose.

Stephen Baker reports grants from Wellcome Trust (Senior Research Fellowships), from Addenbrooke's Charitable Trust, during the conduct of the study.

Michael P. Weekes reports grants from Wellcome Trust (Senior Research Fellowships), from Addenbrooke's Charitable Trust, during the conduct of the study.

\section{References}

1. Rivett L, Sridhar S, Sparkes D, et al. Screening of healthcare workers for SARS-CoV-2 highlights the role of asymptomatic carriage in COVID-19 transmission. eLife 2020. DOI:10.7554/eLife.58728

2. Evans S, Agnew E, Vynnycky E, et al. The impact of testing and infection prevention and control strategies on within-hospital transmission dynamics of COVID-19 in English hospitals. medRxiv 2020. DOI:10.1101/2020.05.12.20095562. 
3. Iacobucci G. Covid-19: Doctors sound alarm over hospital transmissions. BMJ 2020. DOI:10.1136/bmj.m2013

4. Public Health England (PHE). Coronavirus (COVID-19) in the UK. 2020. https://coronavirus.data.gov.uk/ (accessed 23rd May 2020).

5. Shields AM, Faustini SE, Perez-Toledo M, et al. SARS-CoV-2 seroconversion in health care workers. medRxiv 2020. DOI:10.1101/2020.05.18.20105197

6. National Institute for Health and Care Excellence (NICE). COVID-19 rapid guideline: haematopoietic stem cell transplantation. 2020. https://www.nice.org.uk/guidance/ng164/chapter/6-Supporting-staff-includingstaff-who-are-self-isolating (accessed 23rd May 2020).

7. Treibel TA, Manisty C, Burton M, et al. COVID-19: PCR screening of asymptomatic health-care workers at London hospital. The Lancet 2020. DOI:10.1016/s0140-6736(20)31100-4

8. Meredeth LW, Hamilton WL, Warne B, et al. Rapid implementation of real-time SARS-CoV-2 sequencing to investigate healthcare associated COVID-19 infections. medRxiv 2020. DOI:10.1101/2020.05.08.20095687

9. He X, Lau EHY, Wu P, et al. Temporal dynamics in viral shedding and transmissibility of COVID-19. Nat Med 2020. DOI:10.1038/s41591-020-0869-5

10. Grassly NC, Pons-Salort M, Parker EPK, et al. Role of testing in COIVD-19 control. Imperial College London 2020. DOI:10.25561/78439

11. Sridhar S, Forrest S, Kean I, et al. A blueprint for the implementation of a validated approach for the detection of SARS-Cov2 in clinical samples in academic facilities. bioRxiv 2020.

12. UK Government. Stay at home advice. 2020. https://www.gov.uk/government/publications/covid-19stay-at-home-guidance/stay-at-home-guidance-for-households-with-possible-coronavirus-covid-19-infection. (accessed 12th June 2020)

13. Korea Centers for Disease Control \& Prevention (KCDC). Findings from Investigation and analysis of repositive cases. 2020. https://www.cdc.go.kr/board/board.es?mid=a30402000000\&bid=0030 (accessed 23rd May 2020).

14. Wölfel R, Corman VM, Guggemos W, et al. Virological assessment of hospitalized patients with COVID2019. Nature 2020. DOI:10.1038/s41586-020-2196-x

15. Bullard J, Dust K, Funk D, et al. Predicting infectious SARS-CoV-2 from diagnostic samples. Clin Infect Dis 2020. DOI:10.1093/cid/ciaa638 